HNO 2019 $\cdot 67: 322-323$

https://doi.org/10.1007/s00106-019-0642-6

(c) Springer Medizin Verlag GmbH, ein Teil von Springer Nature 2019

S. Dazert

Universitäts-Hals-Nasen-Ohrenklinik, St. Elisabeth-Hospital, Bochum, Deutschland

\title{
Digitalisierung - Herausforderung für die HNO-Heilkunde
}

\author{
90. Jahresversammlung der Deutschen \\ Gesellschaft für Hals-Nasen-Ohren- \\ Heilkunde, Kopf- und Hals-Chirurgie, e.V., \\ Berlin, 29. Mai bis 1. Juni 2019
}

Liebe Kolleginnen, liebe Kollegen,

die digitale Transformation unserer Gesellschaft ist in vollem Gange, und die zunehmende Implementierung digitaler Prozesse in das deutsche Gesundheitssystem hat auch die HNO-Heilkunde erreicht und beeinflusst klinische sowie wissenschaftliche Abläufe in erheblichem Maße. Digitalisierung spielt inzwischen eine wichtige Rolle in der Organisation und Dokumentation medizinischer Abläufe sowie auch in diagnostischen und therapeutischen Anwendungen.

Im internationalen Vergleich liegen deutsche Gesundheitseinrichtungen im Hinblick auf digitale Entwicklungen eher auf hinteren Rängen, sodass sofortige und intensive Anstrengungen in diesen Themenfeldern zwingend erforderlich sind. Folgerichtig heißt das Leitthema der diesjährigen 90. Jahresversammlung der Deutschen Gesellschaft für Hals-Nasen-Ohren-Heilkunde, Kopf- und HalsChirurgie „Digitalisierung in der HNOHeilkunde“. In den Referaten, Vorträgen, Postern und Rundtischgesprächen werden unterschiedliche Bereiche zum Kongressthema bearbeitet und wichtige Aspekte für die Weiterentwicklung und Zukunftssicherung unseres Fachgebiets aufgezeigt.

Im Hinblick auf zukünftige Entwicklungen in der Gesundheitsversorgung ist es erforderlich, digitale Kompetenzen sowohl Ärzten, medizinischem Fachpersonal, aber auch Patienten strukturiert $\mathrm{zu}$ vermitteln. Ebenso müssen die sich aus den medizinisch-technischen Fortschritten ergebenden rechtlichen und ethischen Fragen geklärt werden.

》) Es müssen Qualitätsmerkma-
le für die Nutzung des Internets
bei Gesundheitsinformationen
erarbeitet werden

Wichtig in diesem Zusammenhang sind Aspekte der elektronisch-medizinischen Datenhaltung, die bereits in verschiedene Gesundheitsbereiche Einzug gehalten hat. Insbesondere die elektronische $\mathrm{Ge}$ sundheitskarte sowie die elektronische Patientenakte stehen im Sinne einer höheren Versorgungsqualität bei potenziell geringeren Versorgungskosten im Fokus des Interesses. Die Registerbildung hat die Evaluation der Wirksamkeit in der Versorgungsroutine, das Monitoren der Patientensicherheit sowie die ökonomische Evaluation und die Mindestmengenforschung zur Aufgabe. Darüber hinaus verfolgen Register das Ziel, epidemiologische Zusammenhänge und Unterschiede zu beschreiben, die Qualitätssicherung und -verbesserung sowie die klinische Forschung zu unterstützen. 
Im Kontext mobiler Informationsund Kommunikationstechnologie für medizinische Dienstleistungen steht auch bei der Anwendung von Suchmaschinen und Apps die Qualitätsfrage im Vordergrund. So müssen Qualitätsmerkmale für die Nutzung des Internets bei stetig zunehmender Bereitstellung von Gesundheitsinformationen erarbeitet werden. Für Anwender medizinischer Apps sind Hinweise erforderlich, diese Applikationen nach definierten Qualitätskriterien in verschiedenen Kategorien, wie Funktionalität, Wissenschaftlichkeit, aber auch Datenschutz, zu beurteilen.

Als Beispiel einer unmittelbaren Anwendung für die digital unterstützte Patientenversorgung im Krankenhaus kann die Etablierung eines digitalen Operationssaals der Zukunft angesehen werden. Hier geht es nicht nur um die technische Verbesserung der einzelnen computergestützten Geräte und Instrumente, sondern vielmehr um deren dynamische Vernetzung und Systemintegration in einem offenen modularen System mit dem Ziel, die Anwenderfreundlichkeit, die Qualität und die Patientensicherheit zu verbessern.

Ähnliche Zielsetzungen im Hinblick auf die Versorgungsqualität ergeben sich für die digitalen Entwicklungen der Telemedizin in unserem Fachgebiet sowie für moderne Konzepte der Hörrehabilitation, wo der Einsatz von „artificial intelligence“ und „remote care“ neue Strategien eröffnet.

\section{》) Digitale Lehrstrategien können didaktische Konzepte verstärkt auf den Lernenden zentrieren}

Als Beispiel der digitalen translationalen Forschung kann die molekulargenetische Diagnostik von Hörstörungen in einem Krankheitskontext genannt werden, die heute wesentlich auf der sich rasch entwickelnden Genomik basiert. Der Begriff „big data“ spielt hier eine entscheidende Rolle und beinhaltet die Verwendung großer Datenmengen, die in Form von genetischen Datenbanken,
In-silico-Analysewerkzeugen und Allelhäufigkeitsdatenbanken vorliegen.

In der Nachwuchsförderung sind neue digitale Lehrstrategien von großer Bedeutung. Sie beruhen wesentlich auf technologischen Fortschritten, aber v. a. auf der Motivation, didaktische Konzepte verstärkt auf den Lernenden zu zentrieren. Dieser Ansatz bedeutet gerade für HNO-Heilkunde eine wichtige Herausforderung und Chance.

Berlin ist immer eine Reise wert! Ich freue mich auf ein Wiedersehen und interessante Diskussionen auf unserem Kongress im Mai dieses Jahres.

Herzlichst Ihr

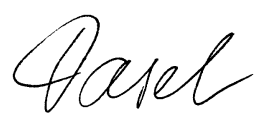

Prof. Dr. Stefan Dazert

Präsident der Deutschen Gesellschaft für Hals-Nasen-Ohren-Heilkunde, Kopfund Hals-Chirurgie e. V., Bonn.

\section{Korrespondenzadresse}

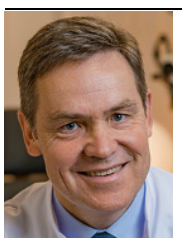

Prof. Dr. med. S. Dazert

Universitäts-Hals-NasenOhrenklinik, St. ElisabethHospital Bleichstr. 15, 44787 Bochum, Deutschland stefan.dazert@rub.de

Interessenkonflikt. S. Dazert gibt an, dass kein Interessenkonflikt besteht.

\section{Leitthemenübersicht HNO 2018-2019}

\section{HNO}

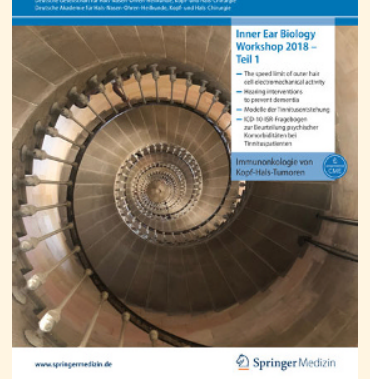

HNO bietet Ihnen umfassende und aktuelle Beiträge zu interessanten Themenschwerpunkten aus allen Bereichen der Hals-, Nasen- und Ohrenheilkunde, Kopf- und Halschirurgie sowie Allergologie und ihren verwandten Gebieten.

\section{Halbjahr 2018 \\ - 07/18 Dysphagie \\ - 08/18 Originalien \\ - 09/18 Originalien \\ - 10/18 Orbita - Teil 1 \\ - 11/18 Orbita - Teil 2 \\ - 12/18 Post-ASCO 2018}

\section{Halbjahr 2019}

- 01/19 Originalien

- 02/19 Originalien

- 03/19 Inner Ear Biology Meeting - Teil 1

- 04/19 Preisträger

- 05/19 Digitalisierung

- 06/19 Inner Ear Biology Meeting - Teil 2

(Änderungen vorbehalten)

Einzelne Ausgaben können Sie direkt bei unserem Kundenservice zum Preis von je EUR 42,- zzgl. Versandkosten beziehen. So erreichen Sie unseren Kundenservice:

Springer Customer Service Center GmbH Kundenservice Zeitschriften Tiergartenstr. 15, 69121 Heidelberg

Tel.: +49 $6221345-4303$

Fax: +49 $6221345-4229$

leserservice@springer.com

www.springermedizin.de/hno-zeitschrift 